\title{
Melatonin and gestational hypertension
}

\author{
Yasuyuki Nagasawa' ${ }^{1}$ Masayoshi Nanami ${ }^{1} \cdot$ Takahiro Kuragano $^{1} \cdot$ Masaharu Ishihara $^{1}$
}

Received: 31 May 2021 / Revised: 14 July 2021 / Accepted: 14 July 2021 / Published online: 12 August 2021

(C) The Japanese Society of Hypertension 2021

\section{Multiple functions of melatonin beyond sleep}

Melatonin is one of the most important elements in the circadian rhythm governed by the suprachiasmatic nucleus and is mainly stimulated by light exposure and food intake [1]. Melatonin has been considered to have an indirect relationship with cardiovascular events via both the duration and quality of sleep, which are both controlled by melatonin. Recent studies have revealed roles of melatonin beyond sleep, such as antihypertension, anticancer, antiapoptotic, and antiplatelet effects (Fig. 1) [2]. These multiple functions of melatonin could be caused directly by melatonin, since melatonin receptors are present in many organs, such as the brain, retina, cardiovascular system, cardiac ventricular wall, aorta, coronary and cerebral arteries, liver, gallbladder, duodenal enterocytes, colon, cecum, appendix vermiformis, skin, parotid gland, exocrine pancreas, kidneys, immune cells, platelets, brown and white adipocytes, breast and prostate epithelial cells, ovaries, granulosa cells, myometrium, placenta, and fetal kidneys [3]. Moreover, melatonin receptors have several variations, such as melatonin receptor type 1a (MT1), melatonin receptor type 1b (MT2), retinoid-related orphan nuclear hormone receptor, and melatonin-related orphan receptor, which could contribute the multiple functions of melatonin. However, the precise functions of all these receptors remain unclear [3]. Blood pressure, sodium excretion from the kidney, and proteinuria are usually affected by the circadian rhythm, so they are also considered to be indirectly controlled by melatonin via sleep. Since salt cannot be consumed while sleeping, salt excretion by the kidney is reduced, and blood pressure

Yasuyuki Nagasawa

nagasawa@hyo-med.ac.jp

1 Division of Kidney, Dialysis and Cardiology, Department of Internal Medicine, Hyogo College of Medicine,

Nishinomiya, Hyogo, Japan decreases at night [4]. However, a recent study revealed that reduced salt excretion might be directly caused by melatonin, resulting in changes to the circadian rhythm of blood pressure [5]. Moreover, melatonin was recently reported to have a direct vascular dilation effect, resulting in blood pressure-lowering effects systemically [2]. These new findings indicate that melatonin is a key player with direct health benefits rather than a secondary player acting only through sleep [4].

\section{Melatonin and pregnancy}

In terms of pregnancy, melatonin has been considered to have an indirect relationship with a healthy continuation of pregnancy via sleep because good sleep contributes to good health in pregnant women (Fig. 2). Many herbivores deliver infants at night, and the time of delivery of humans is also predominantly at night. Pregnancy has a strong relationship with sex hormones, which have a circadian rhythm. These phenomena implicate that there is a direct relationship between pregnancy and melatonin (Fig. 2). Indeed, it was reported that melatonin produced in the ovary and placenta influenced fetal growth [6]. During delivery, the maternal melatonin rhythm is augmented to induce uterine contractility [7]. There are several serious complications that can occur during the late phase of pregnancy, such as preeclampsia, eclampsia, and HELLP syndrome. These complications are accompanied by gestational hypertension, and gestational hypertension induces endothelial dysfunction and coagulation reactions, which cause serious conditions $[7,8]$. Recent studies have revealed the mechanism of these complications and their many related factors $[8,9]$, but at this point, the most effective therapy for these complications is termination. Melatonin remains a therapeutic nontarget for complications in the late phase of pregnancy. However, melatonin might have a direct or indirect relationship with the stabilization of pregnancy and fetal development since melatonin may have a relationship with blood pressure, including gestational hypertension. 


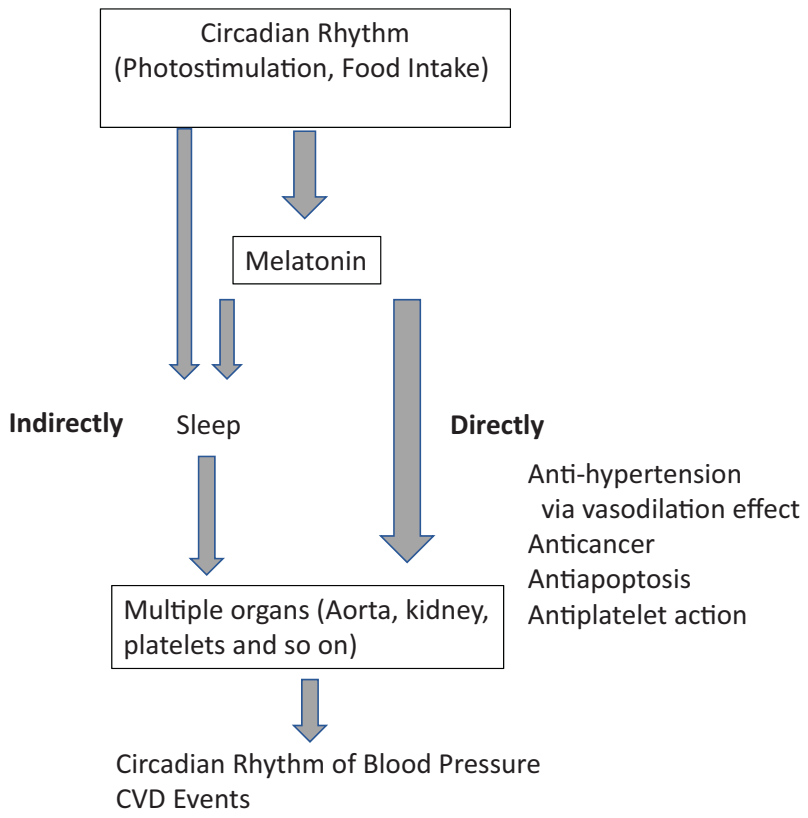

Fig. 1 Relationships between the circadian rhythm and CVD events, including hypertension, through melatonin. The circadian rhythm, governed by the suprachiasmatic nucleus in the brain, is mainly regulated by photostimulation and food intake. According to the circadian rhythm, melatonin is secreted into the brain as well as the systemic bloodstream. Melatonin induces sleep, whereby it indirectly protects against CVD events and hypertension. However, recent findings revealed direct melatonin functions, such as antihypertension, anticancer, and antiapoptotic effects, which might directly protect against CVD events and hypertension

\section{Possibility of targeting melatonin for late- phase complications of pregnancy, including gestational hypertension}

Sun et al. originally established a gestational hypertension mouse model, and melatonin supplementation ameliorated hypertensive phenotypes in these mice. It also enhanced the uterine artery endothelial response to acetylcholine via the BKCa potassium channel [10], which was suppressed by the development of preeclampsia. They found that melatonin improved pregnancy outcomes in mice, as evaluated by the fetal live ratio, fetal weight, and placental weight. Melatonin has been considered to have an indirect relationship with the safety of pregnancy through good sleep. However, these new findings suggest that melatonin could directly contribute to the stabilization of blood pressure during pregnancy (Fig. 2). Drug safety is highly important during pregnancy because the drug should be safe for both mother and fetus. Therefore, drugs for gestational hypertension, eclampsia, and HELLP syndrome are limited, and it is difficult to evaluate the safety of the drugs clinically. As melatonin is an endogenous hormone that has been used as a hypnotic drug, melatonin is expected to be able to successfully treat serious

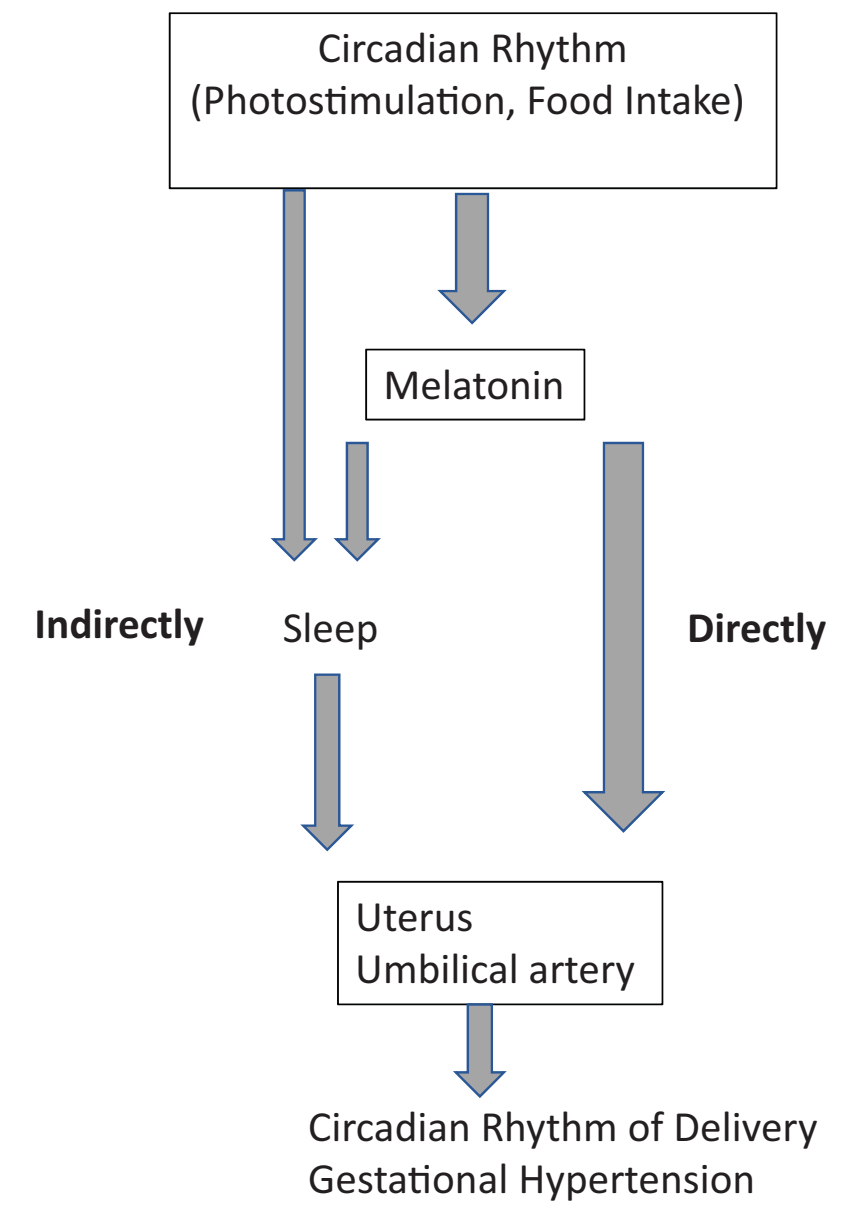

Fig. 2 Relationship between the circadian rhythm and pregnancy, including gestational hypertension and melatonin. According to the circadian rhythm, melatonin is secreted into the brain as well as the systemic bloodstream. Melatonin induces sleep, which indirectly contributes to the circadian rhythm of labor and the stability of blood pressure during pregnancy. However, recent findings indicated that melatonin could directly ameliorate gestational hypertension, resulting in improvement of pregnancy outcomes

complications during pregnancy. There are many unresolved details about the use of melatonin as a therapeutic drug for gestational hypertension. First, the dose and the number of doses of melatonin to treat gestational hypertension should be evaluated. At this point, melatonin has been evaluated only as a sleep inducer. Second, the safety of new doses as treatments for gestational hypertension should be evaluated in both mothers and fetuses. Third, the efficacy of melatonin as a remedy for gestational hypertension should be evaluated, although the results in mice are promising. Melatonin and its analogs have become candidate medicinal therapies for gestational hypertension because effective drugs for complications of the late phase of pregnancy are limited.

Acknowledgements This work was supported by a research grant for rare and intractable disease from the Ministry of Health, Labour and 
Welfare of Japan (20FC1008). This work was supported by a research grant for rare and intractable disease from the Ministry of Health, Labour and Welfare of Japan (20FC1008). This work was also supported by JSPS KAKENHI grant numbers JP17K09721, JP19K10098, JP20K10225 and JP21K08242 and AMED grant numbers JP20ek0109479 and JP21ek0109479.

\section{Compliance with ethical standards}

Conflict of interest The authors declare no competing interests.

Publisher's note Springer Nature remains neutral with regard to jurisdictional claims in published maps and institutional affiliations.

\section{References}

1. Zisapel N. New perspectives on the role of melatonin in human sleep, circadian rhythms and their regulation. $\mathrm{Br} \mathrm{J}$ Pharm. 2018;175:3190-9.

2. Opie LH, Lecour S. Melatonin has multiorgan effects. Eur Heart J Cardiovasc Pharmacother. 2016;2:258-65.

3. Emet M, Ozcan H, Ozel L, Yayla M, Halici Z, Hacimuftuoglu A. A review of melatonin, its receptors and drugs. Eurasia J Med. 2016;48:135-41.
4. Nagasawa Y, Hasuike Y, Kuragano T, Ishihara M. Circadian rhythm and CKD: is melatonin a key player or bi-player? Intern Med. 2019;58:1531-2.

5. Ohashi N, Ishigaki S, Isobe S, Matsuyama T, Sato T, Fujikura T, et al. Salt loading aggravates the relationship between melatonin and proteinuria in patients with chronic kidney disease. Intern Med. 2019;58:1557-64.

6. Voiculescu SE, Zygouropoulos N, Zahiu CD, Zagrean AM. Role of melatonin in embryo fetal development. J Med Life. 2014;7:488-92.

7. Reiter RJ, Tan DX, Korkmaz A, Rosales-Corral SA. Melatonin and stable circadian rhythms optimize maternal, placental and fetal physiology. Hum Reprod Update. 2014;20:293-307.

8. Vangrieken P, Remels AHV, Al-Nasiry S, Bast A, Janssen GMJ, von Rango $\mathrm{U}$, et al. Placental hypoxia-induced alterations in vascular function, morphology, and endothelial barrier integrity. Hypertens Res. 2020;43:1361-74.

9. Colmenares-Mejia CC, Quintero-Lesmes DC, Bautista-Nino PK, Guio Mahecha E, Beltran Avendano M, Diaz Martinez LA, et al. Pentraxin-3 is a candidate biomarker on the spectrum of severity from pre-eclampsia to HELLP syndrome: GenPE study. Hypertens Res. 2020;43:884-91.

10. Sun Y, Wang C, Zhang N, Liu F. Melatonin ameliorates hypertension in hypertensive pregnant mice and suppresses hypertension-induced downregulation of $\mathrm{Ca} 2+$-activated $\mathrm{K}+$ channel in uterine arteries. Hypertens Res. 2021, https://doi.org/ 10.1038/s41440-021-00675-5 